This is a post-peer-review, pre-copyedit version of an article accepted for publication in Synthese. When production is complete, the final authenticated version will be available online at http://dx.doi.org/10.1007/s11229-020-02757-2.

\title{
Paraphrasing Away Properties with Pluriverse Counterfactuals
}

\section{Introduction}

Many philosophers have been attracted to nominalism, the position that everything is concrete, ${ }^{1}$ because abstract objects pose serious philosophical problems. ${ }^{2}$ Nominalism, however, faces a major difficulty. In every essential domain of ordinary inquiry and practice, from particle physics to painting walls, we constantly say things that entail that there are abstract objects. ${ }^{3}$ Nominalists who participate in these activities are constantly in the position of saying things that contradict their nominalism. Nominalists are thus faced with a choice: either abandon engaging in much ordinary inquiry, give up on nominalism, or find some nominalisticallyacceptable replacements for the things we all say in ordinary contexts that entail that there are abstract objects. ${ }^{4}$

In this paper, I offer a "paraphrase" strategy for simple sentences about properties of concrete objects: that is, a strategy for providing nominalistically-acceptable replacements for

\footnotetext{
1 'Nominalism' has been used in other ways. Peter van Inwagen (2011) and L. A. Paul (2017) call my position 'austere nominalism'. For a complete taxonomy, see Michael J. Loux (Ch. 2, 1998).

${ }^{2}$ For example, Paul Benacerraf (1973) raises the epistemological objection to platonism, and Hartry Field (1989) advances it (see especially pp. 25-30). Furthermore, Lewis (1986, Ch. 3, pp. 176-190) gives an argument that "magical ersatz" theories of possible worlds are unintelligible, and Daniel Nolan (Forthcoming) adapts it into a problem for 'instantiates' (understood broadly as predicate applying to both properties and relations). Although Nolan proposes a solution to the problem he raises, I do not think his proposed solution to the problem is successful: he proposes grasping abstract predicates by means of sentences utilizing higher-order quantification, and I doubt that one can understand higher-order quantification without already understanding 'instantiates' or related predicates concerning relations and their relata.

${ }^{3}$ This is assuming that the things we say that seem to be about abstract objects have their apparent entailments. A nominalist might hold that platonist sentences do not have the entailments their surface grammar suggests, much as 'the sun rises every morning' does not entail that the sun moves around the earth. I will set this possibility aside as a concession to platonists.

${ }^{4}$ Van Inwagen (2004) raises this challenge to nominalism about properties, but it has its precedent in the muchdiscussed Quine-Putnam indispensability argument. My response to one is much the same as my response to the other. Field offers a good discussion of the latter argument (pp. 14-20). Note that while I will focus on properties in this paper, I am only addressing the problem of ontological commitment to properties that is carried by quantifying over them and singular terms for them: I am not concerned here with, for example, arguments for their existence based on their utility in explaining resemblance or in accounting for successful predication. Regarding resemblance and predication, for present purposes I am happy to take the standard austere nominalist line and regard these as primitive (see Loux, Ch.3, pp. 52-55 on predication).
} 
them. ${ }^{5}$ In doing so, it makes room for a consistent nominalist worldview while avoiding some serious problems existing paraphrase strategies encounter. ${ }^{6}$ While more needs to be done to provide replacements for all sentences that entail that there are abstract objects, the strategy for paraphrasing these simple sentences has the potential to be extended to accommodate all sentences important for ordinary inquiry and practice that entail that there are abstract objects.

I will first give a problem case for nominalism that is generated by simple sentences about properties of concrete objects. This problem case threatens the above trilemma. I will then give an informal exposition of how the paraphrase strategy is supposed to work. Following this, I will give a more rigorous exposition of the paraphrase strategy. I will then describe how simple sentences about external properties of concrete objects or that contain "external" expressions pose a special challenge for the paraphrase strategy. I will explain how the paraphrase strategy can handle such sentences. Finally, I will conclude by mentioning how the paraphrase strategy might be extended to sentences about different kinds of abstracta.

\section{A Problem Case for Nominalism Generated by Properties of Concrete Objects}

The central problem for nominalism can be illustrated by the following argument:

\section{Argument 1}

(1) Lucy is a female spider and has a prosoma (i.e. combined head and thorax).

(2) Either of two conspecific female spiders has the same anatomical properties as the other.

(3) The property of having a prosoma is an anatomical property.

(4) Every spider is such that it has a prosoma iff it has the property of having a prosoma.

\footnotetext{
${ }^{5}$ An intuitive understanding of simple sentences about properties of concrete objects suffices for most of what follows, but I can be more precise. Simple sentences about properties of concrete objects are sentences that [i] are about properties of concrete objects, [ii] are not about any other abstract objects, [iii] are such that the results of applying steps $1-5$ of the paraphrase strategy $(\S 4)$ to them are nominalistically acceptable and satisfy conditions (a)-(c) and (e) of Pluriverse Preservation ( $\$ 4)$, and [iv] do not contain any expressions linking expressions for properties together (e.g. 'is identical to' in 'some property is identical to some property', 'redness is identical to some property', etc.).

${ }^{6}$ A prominent example is Cian Dorr's (2008) strategy. It can be roughly characterized as follows: sentences like 'A and B instantiate a common property' are replaced with sentences like 'if there were properties, A and B would instantiate a common property'. Dorr concedes that "nominalism is metaphysically necessary if true" (p.37). If Dorr is right, his paraphrases are counterpossibles if nominalism is true. His strategy then controversially requires that counterpossibles are not trivially true (see Timothy Williamson [2017] for a defense of the trivial view). It also requires that 'instantiates' is genuinely meaningful, which is questionable (see fn. 2). Like Dorr's, my paraphrase strategy makes use of counterfactuals, though not ones with impossible antecedents.
} 
(5) Stacie and Lucy are conspecific female spiders.

(C)Therefore, Stacie has a prosoma.

Argument 1 is valid in first-order logic (FOL) under any reasonable translation. Although it begins with a nominalistically acceptable premise to reach a nominalistically acceptable conclusion, it contains premises that are not nominalistically acceptable. Some of the premises entail that properties exist, either individually or in combination with some of the other premises. That there exists an anatomical property is a logical consequence of 'the property of having a prosoma is an anatomical property', and that something has an anatomical property is a logical consequence of 'Lucy is a female spider and has a prosoma', 'the property of having a prosoma is an anatomical property', and 'every spider is such that it has a prosoma iff it has the property of having a prosoma'. ${ }^{7}$ So nominalists are not able to give Argument 1 when being metaphysically serious. Nominalists, then, had better be prepared to offer a replacement, or "paraphrase", for each of Argument 1's nominalistically unacceptable premises if they are to use Argument 1 in ordinary contexts. ${ }^{8}$ Otherwise, they ultimately contradict themselves.

One natural suggestion is to say that nominalists should simply give up on Argument 1 and its nominalistically unacceptable premises altogether, in ordinary contexts and when being

\footnotetext{
7 For present purposes, I assume that 'conspecific' is a primitive predicate. However, some might take the sentences to tacitly quantify over species. On some theories of species, species are nominalistically acceptable entities, so this poses no extra difficulty for the nominalist (see Michael T. Ghiselin [1974] and David L. Hull [1978] for early representatives of the view that species are composed of the organisms belonging to it; see also Berit Brogaard [2004]). Moreover, the paraphrase strategy developed here can be used to avoid committing to species if they are regarded as properties instead (e.g. homo sapiens = the property of being human).

${ }^{8}$ For a similar use of 'paraphrase', see van Inwagen (2004). As I use 'paraphrase', I do not intend for paraphrases to have the same meaning as the original sentences. A paraphrase of a sentence is just a replacement for it that is available when one is pressed to be metaphysically serious. The idea can be made more precise. Let an ideal platonist argument be a valid argument that (i) may but need not have nominalistic premises, (ii) has platonistic premises that are unimpugnable in ordinary life and no other platonistic premises, and (iii) displays crucial ordinary reasoning. A paraphrase strategy is successful for a given language if it replaces every unimpugnable platonistic sentence of that language with a true nominalistic sentence - its paraphrase - and, for every ideal platonist argument of that language, replacing its platonistic sentences how the paraphrase strategy recommends results in an argument such that its conclusion is entailed by its premises and, if needed, some other true nominalistic principles. (Here, a "language" could be a fragment of a larger language.) Paraphrases thus preserve the reasoning important for ordinary affairs while dispensing with everything that is problematic for nominalists.
} 
metaphysically serious. Nominalists are not in any danger of contradicting themselves by refraining from offering the argument and refraining from using some of its premises. Unfortunately for this suggestion, nominalists are not able to give up on Argument 1 in ordinary contexts. Argument 1 and arguments like it enable patterns of reasoning that are crucial in the ordinary affairs of life. The central feature of Argument 1 for ordinary reasoning is that (2)-(4) license one to reason from (1) and (5) to (C) using nothing more than logical principles and very general considerations about anatomy and sameness of sex and species. Being able to reason from (1) and (5) to (C) from very general considerations about anatomy and sameness of sex and species is certainly important for biological investigations. Moreover, Argument 1 can be modified to reach very different conclusions just by making some minor alterations in its components. For instance, 'Stacie' and 'Lucy' can be substituted for names of different things (e.g. 'Suzy' and 'Laura'), and 'prosoma' could be substituted with expressions for other anatomical parts (e.g. 'abdomen', changing 'a' to 'an' where necessary). The resulting arguments would license one to reason about other individuals than Stacie and Lucy and other anatomical parts than prosomas. In short, with Argument 1 one is not only able to engage in crucial ordinary reasoning about Stacie, Lucy, and prosomas, but one is also able to engage in ordinary reasoning about a wide variety of individuals and anatomical parts just by making minor alterations that do not affect its syntactic structure.

Given the role that Argument 1 serves in ordinary reasoning, anyone who wants to replace (2)-(4) to get something nominalistically acceptable needs to be sure that the new premises are related to the same general considerations about anatomy and sameness of sex and species. Moreover, some true general principles, logical or otherwise, must guarantee that (C) is true if (1), (5) and the new premises are. These true general principles must be related to (1), (5), the replacements for (2)-(4), and (C) in such a way that making minor alterations in (1), (5), the replacements for (2)-(4), and (C) in a consistent manner can produce new 
arguments the conclusions of which are guaranteed to be true by these same principles if their premises are true. These arguments must parallel the valid arguments that can be obtained by making consistent alterations in the components of Argument 1 . This is because the utility and versatility of Argument 1 rests not only on [a] the fact that one can apply true general principles to reason from (1)-(5) to $(\mathrm{C})$, but also $[\mathrm{b}]$ the fact that one can apply those same principles to premises syntactically similar to (1)-(5) to reason to conclusions syntactically similar to (C). If the argument that results from replacing (2)-(4) is such that (C) is entailed by (1), (5), and its other premises for overly particular reasons, it will not admit of capturing a plethora of ordinary reasoning just by minor alterations in its parts. This modifiability is one of the most important features of Argument 1.

So, Argument 1 cannot simply be dispensed with. However, there is an obvious nominalist response to it: replace appeals to properties with appeals to the concrete linguistic entities typically thought to correspond to them. ${ }^{9}$ Though this nominalist response fails, considering it is illustrative and lays part of the foundation for a response that succeeds. ${ }^{10}$

Fleshing out the response will require some additional linguistic resources. Let enclosing an inscription on the left and right with tokens of '*' produce a term-token denoting that inscription. Thus $*_{\text {foot }}^{*}$ is the previously occurring token of 'foot' (pretending, if necessary, that this paper is printed out before you). Then if anatomical properties are properties like the property of having a prosoma, the property of having an abdomen, and the property of having eight legs, let anatomical indicators be inscriptions like * $x$ has a prosoma*, *y has an abdomen*, and $*_{z}$ has eight legs*. The enumerated anatomical indicators are meaningful extensions of English. English speakers familiar with the apparatus of first-order

\footnotetext{
${ }^{9}$ Understand all references to concrete linguistic entities below to be references to ones that are singular in meaning. They should exhibit no context-sensitivity or other variability in meaning.

${ }^{10}$ Wilfred Sellars (1963) offers a nominalist system that gives analyses of the sentences about properties in Argument 1 that very loosely resemble the replacements of them I will offer on behalf of the obvious nominalist response. Like the obvious nominalist response, in my estimation Sellars' system cannot adequately handle cases where there are important properties without any corresponding inscriptions.
} 
logic know how to interpret them: tokens of ' $x$ ', ' $y$ ', and ' $z$ ' are occurring instead of 'it'. In fact, *it has a prosoma* could have been included in the list, except that 'it' sometimes functions as what Geach has called a "pronoun of laziness". ${ }^{11}$ Following a convention of van Inwagen (2014), say that a Tarskian language is any language that "consists of closed or open sentences and closed or open terms of English (or some natural language) and the sentential connectives, brackets, quantifiers, variables, and identity-sign of the vocabulary of first-order logic (so-called) with identity—perhaps supplemented by items from the vocabulary of various well-defined extensions of firstorder logic with identity.” (p.1)

For instance, ' $\exists x(x$ has a prosoma)' and ' $\exists y(y$ has a head)' are Tarskian English sentences, and ' $x$ has a prosoma' and ' $y$ has a head' are Tarskian English open sentences. Just as one can speak of Tarskian English sentences and open sentences, so one can speak of Tarskian English sentence-tokens and open sentence-tokens. The anatomical indicators enumerated above are Tarskian English open sentence-tokens, though not all anatomical indicators are. ${ }^{12}$ They are also one-place open sentence-tokens because they each contain exactly one free variabletoken. ${ }^{13}$

With this background in place, one can see how to flesh out the obvious nominalist response. The bulk of the strategy is to replace expressions for anatomical properties with expressions for anatomical indicators and replace verbs and prepositions indicating the possession of a property with verbs and prepositions indicating the satisfaction of an open

\footnotetext{
${ }^{11}$ Geach (1962/1980, p.151).

12 Platonists might reasonably complain that I have not nominalized the notion of Tarskian English. The enumerated anatomical indicators are not intrinsically meaningful: their being meaningful involves some relation to English speakers who understand first-order languages. I will leave nominalizing English, first-order languages, and the relevant semantic and syntactic relations as a further project.

${ }^{13}$ That said, a one-place open sentence-token can have more than one free variable-token provided that every free variable-token in it is a semantic and syntactic duplicate of its first one, as with * $x$ loves $x *$.
} 
sentence-token. In other words, instead of reasoning in terms of having anatomical properties, one should reason in terms of satisfying anatomical indicators. ${ }^{14}$

Taking up this response produces the following, where a prosoma indicator is a oneplace open sentence-token like $* x$ has a prosoma* ${ }^{*} y$ has a prosoma*, etc.:

\section{Argument 2:}

(1) Lucy is a female spider and has a prosoma.

$\left(2_{2}\right)$ Either of two conspecific female spiders satisfies the same anatomical indicators as the other.

$\left(3_{2}\right)$ Every and some prosoma indicator is an anatomical indicator.

$\left(4_{2}\right)$ Every spider is such that it has a prosoma iff it satisfies every and some prosoma indicator.

(5) Stacie and Lucy are conspecific female spiders.

(C) Therefore, Stacie has a prosoma.

Like Argument 1, Argument 2 is valid in first-order logic under any reasonable translation, so some true general principles guarantee that if $(1),(5)$, and $\left(2_{2}\right)-\left(4_{2}\right)$ are true, $(\mathrm{C})$ is true as well. Moreover, valid arguments that parallel the valid arguments obtainable by consistently altering the components of Argument 1 can be obtained by consistently altering the components of Argument 2. Unfortunately, arguments like Argument 2 will not do as replacements for arguments like Argument 1. There almost certainly are not anatomical indicators for every anatomical property: some have not yet been discovered, and many are so complex that it is unlikely anyone will ever produce a corresponding inscription. $\left(2_{2}\right)$ therefore fails to express the full generality of the relationship between sameness of species and sex, on the one hand, and anatomical structure, on the other, that is expressed by 'either of two conspecific female spiders has the same anatomical properties as the other'. Moreover, even if there are anatomical indicators for every anatomical property, this is only because we exist at a lucky moment in history. One would not want the nominalist paraphrase to have been unusable before an inscription for every anatomical property was produced, for then the

\footnotetext{
${ }^{14}$ The tricky part is with what to replace 'the property of having a prosoma', a singular term. I choose to replace it with a phrase indicating universal and existential quantification over open sentence-tokens that solely indicate of things that they have prosomas.
} 
strategy for paraphrasing other cases where there is an appeal made to properties will almost certainly fail in those cases where significant facts are unknown.

The next suggestion is to modify the obvious nominalist response by including modal operators. For example, one might propose paraphrasing (2), (3), and (4) in such a way as to produce the following argument:

\section{Argument 3:}

(1) Lucy is a female spider with a prosoma.

(23) Any two conspecific female spiders are such that, necessarily, if both exist, they satisfy the same anatomical indicators.

(33) Necessarily, every prosoma indicator is an anatomical indicator and possibly, there is a prosoma indicator.

(43) Every spider is such that it has a prosoma iff necessarily, if it exists, it satisfies every prosoma indicator.

(5) Stacie and Lucy are conspecific female spiders.

(C) Therefore, Stacie has a prosoma.

Unlike $\left(2_{2}\right),\left(2_{3}\right)$ expresses the full generality of the relationship between sameness of species and sex and anatomical structure that is expressed by 'either of two conspecific female spiders has the same anatomical properties as the other'. Unfortunately, unlike Argument 2, Argument 3 is not a logically valid argument. By itself, this is not an insurmountable problem: if some true general principles could be found that entail (C) when conjoined with (1), (5), and (23)(43), that would be enough for the paraphrases to be adequate for the case of Argument 1 . It is unclear what true general principles do this, however. Whatever these true general principles are, they need to be such that paraphrases of sentences about properties developed along the above lines always yield replacement arguments the premises of which entail the conclusions of the original arguments when conjoined with the principles (provided the originals are valid). Argument 1 is only an illustrative example, after all: there are many more arguments with premises about properties, and one wants paraphrases of their nominalistically-objectionable premises too. The difficulty is compounded by the fact that it is unclear what the algorithm for producing paraphrases is on the modified strategy. How should, for example, 'every spider $x$ 
is such that there is an anatomical property $y$ such that there is a spider $z$ such that there is an anatomical property $v$ such that $x$ has $y$ and does not have $v$ and $z$ has $v$ and does not have $y^{\prime}$ be paraphrased under this strategy?

Nevertheless, these suggestions are on the right track. The trick to a successful nominalist paraphrase is to be able to describe a scenario in which the realist about properties would say that every anatomical property is expressed by some open sentence-token or other, but to make the description without overtly relying on properties. The nominalist can then use counterfactuals about this scenario to produce a successful replacement for Argument 1 and its objectionable components. Some true general principles concerning these counterfactuals, as well as the principles of logic, will guarantee that (C) is true if (1), (5), and the other premises of this replacement argument are. Moreover, these principles will be such that the replacement argument admits of many consistent alterations of its components that enable reasoners to come to new conclusions from new premises by means of the principles. The replacement argument, then, will serve the same function in ordinary reasoning as Argument 1. In addition, the nominalist paraphrase strategy and the true general principles to be introduced will extend far beyond Argument 1 in their application. ${ }^{15}$ I turn now to giving an informal explanation of what the paraphrase strategy to be developed is attempting to do before giving a rigorous presentation of the strategy.

\section{Informal Exposition of the Paraphrase Strategy}

Let a universe be a composite of some things that (i) are all spatiotemporally related to one another, and (ii) are such that none of them are spatiotemporally related to anything that isn't

\footnotetext{
${ }^{15}$ For another nominalist system that appeals to possibilities for open sentence-tokens, see Charles Chihara (1990). Chihara uses "constructibility quantifiers" and facts about what open sentence-tokens can be constructed to provide a nominalist foundation for mathematics. I object to Chihara's constructibility quantifiers, but I suspect that his system could be modified along the lines of my proposal by making it exploit facts about what open sentence-tokens would exist were there a pluriverse (see §4) rather than exploit facts about what open sentence-tokens can be constructed. Demonstrating this is a task for another time.
} 
one of them. ${ }^{16}$ Suppose that in addition to our universe - the universe containing everything to which we are spatiotemporally related - there are numerous other universes. These universes are so qualitatively diverse that every expression of every possible language is tokened by some inscription or other. ${ }^{17}$ Then for every property of concrete objects, there is a one-place open sentence-token that expresses it: every property of concrete objects is such that in some possible language there is a one-place open sentence that expresses it, and every expression is tokened. ${ }^{18}$ It turns out that the obvious nominalist response is correct after all. Instead of quantifying over anatomical properties, one can quantify over anatomical indicators. Every anatomical property really does have an associated anatomical indicator, but many of them are not parts of our universe. Argument 2 is a good replacement for Argument 1.

Unfortunately, there aren't all these other universes, or at least there is no convincing reason to believe that there are. The probability that there are such universes is an incredibly small fraction of 1 . It is, however, possible for there to be all these universes (a point I will

\footnotetext{
${ }^{16}$ Universes match Lewis' (1986, Ch. 1) view of possible worlds. Unlike Lewis, I don't use universes to analyze possibility.

${ }^{17}$ Or rather, all expressions corresponding to qualitative properties of concrete objects are tokened, plus some expressions corresponding to non-qualitative properties as indicated in fn. 18. Kaplan's-Paradox-like considerations reveal that if every property is expressible, it's impossible for all possible expressions to be jointly tokened. Necessarily, if some token exists, there are more non-qualitative properties concerning pluralities of tokens (e.g. being among the tokens $x, y$, and $z$; not being among the tokens $x, y$, and $z$ ) than there are tokens. Happily, only those non-qualitative properties that can be expressed by tokens created via the method described in fn. 18 are of importance, and the possibility of those tokens existing alongside tokens that express every qualitative property does not result in contradiction.

${ }^{18}$ Or rather, for every important such property. I assume that every qualitative property of concrete objects can be expressed by some token. Moreover, it is possible for non-qualitative properties of concrete objects belonging to one universe (e.g. the property of being identical to Cleopatra) to be expressed by tokens in other universes: all that is needed is for there to be some universe $U$ in which there is a description of the first universe down to the microscopic level, followed by the introduction of descriptions for the individuals the nonqualitative properties concern. Names can then be affixed to the individuals through the descriptions in $\mathrm{U}$, and open sentences involving those names can then be tokened in U. For example, an immortal being or eternallyrunning machine with a vocalization apparatus might declare "there is a universe such that... [fill in a description uniquely satisfied by our universe]. Let ' $A$ ' be a name for the thing in that universe such that [fill in a description uniquely satisfied by Cleopatra in our universe]" and then inscribe the sentence ' $x$ is identical to A'. The resulting open sentence-token then expresses the property of being identical to Cleopatra. (See Neil Sinhababu [2008, pp. 255-256] for a similar point concerning de re thought.) Note that this suggestion does not entail that every non-qualitative property of concrete objects is expressible by tokens: no gerrymandered, uncountable plurality of concrete things is such that any token expressing the property of belonging to it could be generated through even an idealized extension of this process.
} 
return to later). As a first pass, one might try to get a replacement for Argument 1 by using sentences that describe the class of possible worlds in which there are such universes but our universe is not intrinsically any different from how it is in fact: black widows longer than 0.7 inches are still longer than 0.7 inches, Neil Armstrong is still the first person to have landed on the moon, the number of giant squids inhabiting the depths of the ocean is the same, etc. Since our universe is not intrinsically any different in these worlds from how it is in fact, one can read backwards from facts about which parts of our universe satisfy what open sentence-tokens in these worlds to facts about how they actually are. For instance, if it is the case that in each of these possible worlds (a) either of two conspecific female spiders that belong to our universe satisfy the same anatomical indicators as the other, and (b) every and some prosoma indicator is an anatomical indicator, then one can be sure that in each of these possible worlds, any two female black widows belonging to our universe either both have prosomas or neither do. Moreover, given that in each of these possible worlds, female black widows belonging to our universe are not intrinsically any different from how they are in fact, one can be sure that if in each of these possible worlds, any two female black widows belonging to our universe either both have prosomas or neither do, then any two female black widows belonging to our universe either both have prosomas in fact or neither do in fact. One could then reason ordinarily using sentences about these possible worlds.

The problem with this first pass is that possible worlds are not nominalistically acceptable, and nominalists cannot appeal to possible languages to circumscribe the possibilities they are interested in. ${ }^{19}$ Nominalists can, however, give a nominalistic analog of the above strategy: one that functions the same way, but does not require appealing to anything

\footnotetext{
${ }^{19}$ Abstract possible worlds aren't, anyway. There aren't enough universes to serve as concrete possible worlds, but if there were, the obvious nominalist response would be adequate.
} 
nominalists can't accept. With this basic picture of what the nominalist paraphrase strategy to be articulated is attempting to do, I turn to the paraphrase strategy itself.

\section{The Paraphrase Strategy in Detail}

In order for my paraphrase strategy to be developed, I will introduce a few key expressions. The first is 'the actual things', a plural term that rigidly designates everything that in fact exists. The next expression that I will introduce is 'worldly'. 'Worldly' is an attributive adjective. Its function is given by the following schema, where ' $\Psi$ ' is a schematic letter for a common noun: $x$ is a worldly $\Psi:=x$ is (a/an) $\Psi$ and $x$ is one of the actual things.

For example, 'Suzy is a worldly grasshopper' means that Suzy is a grasshopper and one of the actual things, 'Sarah is a worldly violinist' means that Sarah is a violinist and one of the actual things, etc. The final expression to be introduced is the common noun 'pluriverse'. Speaking loosely, pluriverses can be described as things that (i) are composed of universes, and (ii) are such that for every way a universe could possibly be, one of its universe parts is that way. A pluriverse is like Lewis' (1986) plurality of worlds, except that possibility is not analyzed in terms of it. ${ }^{20}$ That informal description is not nominalistically acceptable, however. The nominalistic definition of 'pluriverse' is:

$x$ is a pluriverse $:=x$ is composed of some $y$ s such that...

a. each of the $y$ s is a universe, and

b. the $y$ s are as varied as it is possible for any universes to be.

Since universes are necessarily causally isolated from each other - a fact that will be discussed in greater detail later on - and do not occupy a common space, they do not constrain each other either in number or in qualitative variation. Pluriverses, then, have a vast quantity of extremely diverse universes as parts. If it is possible for there to be a universe with talking caterpillars, then pluriverses must contain some universe with talking caterpillars; if it is possible for there to be a universe composed of dragons, then pluriverses must contain some universe composed

\footnotetext{
${ }^{20}$ For related but different uses of 'pluriverse', see Sider (2002) and José A. Benardete (1964, pp. 149-154).
} 
of dragons; and so on. Because of the enormous extent of qualitative variation possible among universes, pluriverses must contain infinitely many of them (and an impressive infinity at that). Moreover, aside from expression-tokens that have parts that refer to merely possible individuals, there can't be expression-tokens that differ in meaning from every expressiontoken part of a pluriverse.$^{21}$ Informally: the possibilities regarding the meaning of expressiontokens are components of the ways universes can be, so if some universes realize the latter, their expression-token parts must realize the former.

It is important to note that pluriverses are metaphysically possible, which ensures that my paraphrases do not invoke an impossible scenario. I have already remarked that universes do not constrain each other in number or qualitative variation. So long as it's possible for there to be at least two universes, then, a pluriverse is possible: there is no interaction between universes to bar the possibility of a pluriverse. The only question is whether it's possible for there to be spatiotemporal things that are not spatiotemporally connected, which is both necessary and sufficient for there to be at least two universes. But surely it is. It seems entirely possible for there to be a chair, a tree, a rock, or an atom that is not at any distance in space or time from me even as I live and breathe. So it is possible for there to be at least two universes, and since universes in no way constrain one another, it is possible for there to be a pluriverse.

I am now ready to give the paraphrase strategy. I will give it in a series of steps, alongside some commentary when necessary to explain their meaning. I will also show how an example sentence is to be paraphrased: 'there is at least one anatomical property that every spider has and the property of having ten legs is not such an anatomical property'. The first step is:

\footnotetext{
${ }^{21}$ See fn. 18 to see how the pluriverse captures de re content about objects within universes. For every object composed out of parts that exist in multiple universes, I assume that if possibly, there is a pluriverse, it exists, and it is not referred to by any expression-token, then necessarily, it is not referred to by an expression-token. As for merely possible individuals, it is plausible that they cannot be referred to until they come into being, and even the existence of a pluriverse does not guarantee that every possible individual is brought into existence.
} 
- Put any sentence in which there is a common noun for properties or a singular term for a property as the consequent of a counterfactual with the antecedent 'there is a pluriverse'.

Thus 'there is at least one anatomical property that every spider has and the property of having ten legs is not such an anatomical property' becomes 'there is a pluriverse $\square \rightarrow$ there is at least one anatomical property that every spider has and the property of having ten legs is not such an anatomical property', where ' $\square \rightarrow$ ' abbreviates 'if it were the case that..., it would be the case that...' and italics indicates a change from the original sentence dictated by the step. The second step is:

- For every counterfactual with the antecedent 'there is a pluriverse' that results from the previous step, replace every common noun for concreta with the common noun that results from concatenating 'worldly' with it everywhere it occurs in the consequent except where it occurs inside of expressions for properties.

For instance, the common nouns 'donkey', 'electron', and 'car' would be replaced with 'worldly donkey', 'worldly electron', and 'worldly car'. Moving on from the previous step, 'there is a pluriverse $\square \rightarrow$ there is at least one anatomical property that every spider has and the property of having ten legs is not such an anatomical property' becomes 'there is a pluriverse $\square \rightarrow$ there is at least one anatomical property that every worldly spider has and the property of having ten legs is not such an anatomical property'. Note that 'legs' is not replaced with 'worldly legs' because it occurs inside a singular term for a property and that 'pluriverse' is not replaced with 'worldly pluriverse' because 'pluriverse' occurs in the antecedent. The third step is:

- Replace common nouns for properties in the results of the previous step with the corresponding common nouns for one-place open sentence-tokens, both worldly and 
non-worldly, everywhere common nouns for properties occur except where they occur inside of singular terms.

The idea of one-place open sentence-tokens corresponding to properties was discussed earlier. In truth, nominalists do not think that one-place open sentence-tokens correspond to properties at all, for they do not believe that there are properties to be corresponded to. Nevertheless, nominalists and realists about properties both agree that if there are properties, anatomical indicators correspond to anatomical properties. Based on that agreement, one can say that 'anatomical indicator' is a common noun for one-place open sentence-tokens that corresponds to 'anatomical property'. They correspond in the sense that nominalists and realists agree that if there are properties, the things for which the former common nouns are common nouns correspond to the things for which the latter common nouns are common nouns. The same point can be made for 'chemical property' and 'chemical indicator', 'psychological property' and 'psychological indicator', etc., where the indicator common nouns pick out just the inscriptions one would expect them to pick out (e.g. * $x$ is composed of oxygen and hydrogen atoms*, $* x$ is in pain*). Taking the example sentence from the previous step, 'there is a pluriverse $\square \rightarrow$ there is at least one anatomical property that every worldly spider has and the property of having ten legs is not such an anatomical property' becomes 'there is a pluriverse $\square \rightarrow$ there is at least one anatomical indicator that every worldly spider has and the property of having ten legs is not such an anatomical indicator'.

The fourth step is as follows:

- Replace singular terms for properties in the results of the previous step with phrases composed of the corresponding common nouns for one-place open sentence-tokens and an expression indicating universal and existential quantification.

For instance, 'the property of being composed of oxygen and hydrogen atoms' becomes 'every and some oxygen-and-hydrogen-composition indicator', 'the property of being in pain' 
becomes 'every and some pain-possession indicator', and so on. Just as one can speak of common nouns for properties corresponding to common nouns for one-place open sentencetokens, so one can speak of singular terms for properties corresponding to such common nouns: the rationale is essentially the same. The sentence from the previous step goes from 'there is a pluriverse $\square \rightarrow$ there is at least one anatomical indicator that every worldly spider has and the property of having ten legs is not such an anatomical indicator' to 'there is a pluriverse $\square \rightarrow$ there is at least one anatomical indicator that every worldly spider has and every and some tenleg indicator is not such an anatomical indicator', where a ten-leg indicator is an open sentencetoken like $* x$ has ten legs $*{ }^{*} y$ has ten legs*, etc.

This leads to the fifth step, which is

- Replace 'instantiates', 'exemplifies', 'has' where it occurs synonymously with 'instantiates', etc., and their grammatical variants in the results of the previous step with 'satisfies' and its appropriate grammatical variants.

By the fifth step, 'there is a pluriverse $\square \rightarrow$ there is at least one anatomical indicator that every worldly spider has and every and some ten-leg indicator is not such an anatomical indicator', the result of the previous step, becomes 'there is a pluriverse $\square \rightarrow$ there is at least one anatomical indicator that every worldly spider satisfies and every and some ten-leg indicator is not such an anatomical indicator'. Following these five steps has now produced a nominalisticallyacceptable sentence. ${ }^{22}$

\footnotetext{
${ }^{22}$ It is worth remarking that the ideology of the paraphrases is only modestly more complex than the ideology involved in property discourse. Where property discourse involves 'instantiates', the paraphrases have 'satisfies'; where property discourse has 'anatomical property', the paraphrases have 'anatomical indicator'; etc. The ideological additions that lack a platonic parallel are the counterfactual operator and 'are as varied as it is possible for any universes to be', which is used in defining 'pluriverse'. But while platonists could analyze the counterfactual operator in terms of a nearness relation between possible worlds and define 'pluriverse' in terms of ways universes can possibly be, the addition of two ideological primitives is a small price to pay for avoiding the extremely abundant ontology of platonism.
} 
One can now ask what following these five steps yields with respect to Argument 1.

The result is the following argument:

\section{Argument 1*}

(1) Lucy is a female spider and has a prosoma.

$\left(2^{*}\right)$ There is a pluriverse $\square \rightarrow$ either of two worldly conspecific female spiders satisfies the same anatomical indicators as the other.

(3*) There is a pluriverse $\square \rightarrow$ every and some prosoma indicator is an anatomical indicator.

(4*) There is a pluriverse $\square \rightarrow$ every worldly spider is such that it has a worldly prosoma iff it satisfies every and some prosoma indicator.

(5) Stacie and Lucy are conspecific female spiders.

(C) Therefore, Stacie has a prosoma.

At this point two questions must be asked about Argument $1^{*}$ : (A) do some true general principles guarantee that the conclusion is true if the premises are true?, and (B) does Argument $1^{*}$ license the same patterns of reasoning essential for ordinary endeavors that Argument 1 licenses, both individually and via substitution of components of their sentences? If the answer to (A) and (B) is yes, then the procedure has adequately generated paraphrases of (2)-(4), or at least it has with respect to their function in the reasoning associated with Argument 1.

In order to answer (A) affirmatively, some general principles must be introduced to supplement the ones familiar from first-order logic. The first is:

Counterfactual Entailment $(\mathrm{CE})$ : for any sentences $\mathrm{P}, \mathrm{Q}_{1}, \mathrm{Q}_{2}, \ldots, \mathrm{Q}_{\mathrm{n}}$, and $\mathrm{R}$, if $\left(\mathrm{Q}_{1}, \mathrm{Q}_{2}\right.$, $\ldots, \mathrm{Q}_{\mathrm{n}} \vdash$ FoL $\left.\mathrm{R}\right)$ and ${ }^{ } \diamond \mathrm{P}^{\mathrm{r}}$ is true, then ${ }^{\mathrm{r}}\left(\left(\mathrm{P} \square \rightarrow \mathrm{Q}_{1}\right) \wedge\left(\mathrm{P} \square \rightarrow \mathrm{Q}_{2}\right) \wedge \ldots \wedge\left(\mathrm{P} \square \rightarrow \mathrm{Q}_{\mathrm{n}}\right)\right) \rightarrow(\mathrm{P}$ $\square \rightarrow R)^{\top}$ is true.

For example, CE entails that if 'something is a dog' is derivable from 'Fido is a dog' in FOL and 'possibly, pigs fly' is true, then '(pigs fly $\square \rightarrow$ Fido is a dog) $\rightarrow$ (pigs fly $\square \rightarrow$ something is a dog)' is true. Informally, CE amounts to the claim that states of affairs that are logically possible are closer than logically impossible ones. This is because if some logically impossible worlds are closer than logically possible ones, then even if $\mathrm{P}$ is possible, there's no guarantee that classical logic holds at the nearest P-world. In that case, there's no guarantee that ${ }^{r}((\mathrm{P} \square \rightarrow$ 
$\left.\left.\mathrm{Q}_{1}\right) \wedge\left(\mathrm{P} \square \rightarrow \mathrm{Q}_{2}\right) \wedge \ldots \wedge\left(\mathrm{P} \square \rightarrow \mathrm{Q}_{\mathrm{n}}\right)\right)^{\urcorner}$is true only if ${ }^{\ulcorner}(\mathrm{P} \square \rightarrow \mathrm{R})^{\urcorner}$is true despite the fact that $\left(\mathrm{Q}_{1}\right.$,

$\left.\mathrm{Q}_{2}, \ldots, \mathrm{Q}_{\mathrm{n}} \vdash \mathrm{FOL} \mathrm{R}\right)$. The second principle is:

Pluriverse Preservation (PP): for any sentences $\mathrm{P}$ and $\mathrm{Q}$ that

a. do not contain words composed of quantifiers and common nouns (e.g. 'everything' and 'something', as opposed to 'every' and 'some'),

b. do not contain common nouns that categorize non-concrete things,

c. do not contain terms for non-concrete things,

d. only contain expressions the application of which to particular things depends solely on how those things are intrinsically and how they are related to one another, and

e. do not contain any non-truth-functional operators that block existential commitment to the things within their scope, such as 'possibly', 'believes that', or 'according to the fiction',

if $\mathrm{P}$ and $\mathrm{Q}$ differ only in that inside $\mathrm{Q}$, every common noun in $\mathrm{P}$ is replaced wherever it occurs in $\mathrm{P}$ with the result of concatenating 'worldly' with that common noun, then ' $[\mathrm{P}$ $\leftrightarrow$ (there is a pluriverse $\square \rightarrow Q$ Q) ] is true. ${ }^{23}$

For example, PP entails that 'some dog is happy $\leftrightarrow$ (there is a pluriverse $\square \rightarrow$ some worldly dog is happy)' is true. Informally, PP amounts to asserting that however the actual things are in fact, intrinsically speaking, is how they would be were there a pluriverse. Except for (d), the restrictions (a)-(e) are to rule out problem cases that are irrelevant to simple sentences about properties of concrete objects; (d) will be discussed in more detail later, as simple sentences that violate (d) require some special care. Note that no sentence in Argument $1 *$ violates (d).

At this point two things must be done. One is that it must be established that these principles are true. The second is it must be established that these principles guarantee, alongside the principles of logic, that the conclusion of Argument $1 *$ is true if its premises are true. I will first establish this second point, then turn to the first.

Consider first premise (1) and the sentence 'Lucy is a worldly female spider and has a worldly prosoma'. (1) and the latter sentence differ only in that in the latter sentence, every common noun in (1) is replaced everywhere it occurs in (1) with the result of concatenating 'worldly' with that common noun. Consequently, by PP, the following sentence is true: 'Lucy

\footnotetext{
${ }^{23}$ Note that this is intended to include trivial cases, e.g. where $P$ and $Q$ are both 'Tom is excitable', or are both 'Sarah is resilient', or... etc.
} 
is a female spider and has a prosoma $\leftrightarrow$ (there is a pluriverse $\square \rightarrow$ Lucy is a worldly female spider and has a worldly prosoma)'. The sentence 'there is a pluriverse $\square \rightarrow$ Lucy is a worldly female spider and has a worldly prosoma' is logically derivable from (1) and 'Lucy is a female spider and has a prosoma $\leftrightarrow$ (there is a pluriverse $\square \rightarrow$ Lucy is a worldly female spider and has a worldly prosoma)'. Consequently, if (1) is true, PP and logic guarantee that 'there is a pluriverse $\square \rightarrow$ Lucy is a worldly female spider and has a worldly prosoma' is true. Call this last sentence premise (1.5). Moreover, by parallel reasoning, PP and logic guarantee that 'there is a pluriverse $\square \rightarrow$ Stacie and Lucy are worldly conspecific female spiders' is true if (5) is true. Call this sentence premise (5.5). Now consider another sentence: 'there is a pluriverse $\square \rightarrow$ Stacie has a worldly prosoma'. An important fact about this sentence is that its consequent, 'Stacie has a worldly prosoma', is a logical consequence of the consequents of $(1.5),\left(2^{*}\right)-\left(4^{*}\right)$, and (5.5). Another relevant fact is that 'possibly, there is a pluriverse' is true. Given these two facts, it follows by $\mathrm{CE}$ that the following sentence is true, where '(1.5)', '( $\left.2^{*}\right)$ ', etc., are abbreviations for their corresponding sentences: ' $\left((1.5) \wedge\left(2^{*}\right) \wedge \ldots \wedge\left(4^{*}\right) \wedge(5.5)\right) \rightarrow($ there is a pluriverse $\square \rightarrow$ Stacie has a worldly prosoma)'. And if that sentence, (1.5), (2*)-(4*), and (5.5) are true, conjunction introduction and modus ponens guarantees that 'there is a pluriverse $\square \rightarrow$ Stacie has a worldly prosoma' is true. This leads back to PP. Since 'Stacie has a prosoma' differs from 'Stacie has a worldly prosoma' only in that in the latter, every common noun in the former is replaced everywhere it occurs in the latter with the common noun that results from concatenating 'worldly' with it, one can conclude on the basis of PP that the following sentence is true: 'Stacie has a prosoma $\leftrightarrow$ (there is a pluriverse $\square \rightarrow$ Stacie has a worldly prosoma)'. 'Stacie has a prosoma' is a logical consequence of this last sentence and 'there is a pluriverse $\square \rightarrow$ Stacie has a worldly prosoma'. So it follows from PP and logic that if 'there is a pluriverse $\square \rightarrow$ Stacie has a worldly prosoma' is true, 'Stacie has a prosoma' is true as well. 
'Stacie has a prosoma' is the conclusion, so it has now been shown how PP, CE, and logical principles guarantee that the conclusion of Argument $1 *$ is true if its premises are. ${ }^{24}$

The next point to establish is that $\mathrm{CE}$ and PP are true. It is easy to see that $\mathrm{CE}$ is true: as mentioned earlier, it amounts to the claim that states of affairs that are logically possible are closer than logically impossible ones. CE is entailed by most counterfactual logics for just that reason. ${ }^{25}$ Justifying PP is more demanding, but the rationale behind it is straightforward. PP is true because universes are necessarily causally isolated. No matter how many universes there are, how they are, or how they could have been, no parts of any universe will ever causally interact with any parts of any other universe. Spatiotemporal relatedness is necessary for causal interaction. If universes are necessarily causally isolated, then our universe would not be any different intrinsically if there were a pluriverse. Though there would be a vast plentitude of diverse universes were there a pluriverse, if none of them have any effect upon our universe, there's no reason why, for example, the American Revolution would not have occurred, penicillin wouldn't be discovered by Alexander Fleming in 1928, dinosaurs wouldn't have gone extinct on our planet, or there wouldn't be eight planets in our solar system. The history of our universe would have carried on however it did in fact. ${ }^{26}$

\footnotetext{
${ }^{24}$ The structure of argument displayed here resembles Lukas Skiba's [Forthcoming] adaptation of Richard Woodward's (2010) general proof of inferential safety for fictionalists. Woodward and Skiba interpret the property fiction as Dorr's paraphrase strategy, and their proofs therefore inherit all the same problematic assumptions (see fn. 6) - though Woodward is more sanguine than I or Dorr on the possibility of properties. However, because the structure of argument here so closely resembles Skiba's, it is likely that a very similar general proof of inferential safety could be provided for the paraphrase strategy I am proposing.

${ }^{25}$ For instance, it is a consequence of Lewis' $(2001,1973)$ stronger rule "Deduction within Conditionals" in Counterfactuals (p.132).

${ }^{26}$ One might think that universes are necessarily causally isolated de dicto - i.e. one might think that necessarily, no part of one universe causally interacts with a part of another universe - while denying it de re - i.e. while denying that necessarily, every universe $u$ is such that necessarily, no part of $u$ causally interacts with a part of a universe that is not $u$. For example, if we call our universe Alpha, the de re reading is false if it is metaphysically possible for Alpha to be a part of a larger universe. If such a possibility were to obtain, Alpha would not be a universe at all, but rather a proper part of one. Parts of Alpha could then causally interact with parts of the universe of which Alpha is a part that are not themselves parts of Alpha. I grant that the de re reading might be false, but nonetheless maintain that were there a pluriverse, Alpha would retain its universe status and remain causally isolated. This is because (speaking platonistically) of all the possible worlds that contain a pluriverse, the ones nearest to the actual world are the ones in which the actual things are only spatiotemporally related to each other and thus still compose a universe. Changing what spatiotemporal relations the actual things stand
} 
So (A) can be answered affirmatively. I now turn to (B). I consider first whether Argument $1 *$ individually licenses the same patterns of reasoning displayed in Argument 1 , then consider whether it can be modified in parallel ways to Argument 1 to capture ordinary reasoning in other cases. Argument $1^{*}$ individually licenses the same patterns of reasoning displayed in Argument 1. Just as (2)-(4) license one to reason from (1) and (5) to (C) using nothing more than logical principles and very general considerations about anatomy and sameness of sex and species, so do (2*)-(4*) license one to reason from (1) and (5) to (C) using nothing more than logical principles; CE; PP; and very general considerations about anatomy, sameness of sex and species, and the abundance of anatomical indicators in a pluriverse. Moreover, just as (2)-(4) are appropriate for acceptance in ordinary contexts, so $(2 *)-\left(4^{*}\right)$ are true. $\left(2^{*}\right)$ is true because any two female spiders of the same species are anatomical duplicates, and given [any two conspecific female spiders are anatomical duplicates] and [if there were a pluriverse, our universe would not be intrinsically any different from how it is in fact], it is true that [were there a pluriverse, any two conspecific worldly female spiders would be anatomical duplicates]. ( $\left.3^{*}\right)$ is obviously true. Finally, $\left(4^{*}\right)$ is true because (a) necessarily, every $x$ is such that if there is a prosoma indicator, $x$ has a prosoma iff it satisfies every and some prosoma indicator, and (b) were there a pluriverse, there would be a prosoma indicator and every part of a worldly thing would be worldly. In addition to Argument $1 *$ individually licensing the same patterns of reasoning displayed in Argument 1, Argument $1^{*}$ can also be modified how Argument 1 can be modified in order to capture other cases of ordinary reasoning. Just as in Argument 1, in Argument 1*, 'Stacie' and 'Lucy' can be replaced with 'Suzy' and 'Laura',

\footnotetext{
in, even with respect to concrete objects not among them, reflects a greater departure from what is actually the case than holding those relations fixed. The different possible worlds in which enough and only enough concrete objects are added to the actual things for there to be a pluriverse are qualitatively indistinguishable: the only aspect in which these worlds can differ in their closeness to the actual world is in how similar the actual things are in them compared to the actual world, and composing a universe is a significant dimension of similarity. If the point remains in doubt, the antecedents of the paraphrases could be replaced with 'there is a pluriverse and the actual things are only spatiotemporally related to each other', which guarantees that Alpha remains a universe in the relevant nearby possibilities.
} 
and 'prosoma' can be replaced with 'abdomen'. In fact, the paraphrase of the arguments that result from these substitutions in Argument 1 will be identical to arguments that result from modifying Argument $1 *$ in the exact same way.

So the paraphrase strategy has given us, in Argument $1^{*}$, a good nominalistically acceptable replacement for Argument 1. It must be asked, though, how well the paraphrase strategy performs with other arguments that are important for ordinary affairs and contain simple sentences about properties of concrete objects. It does well. For instance, it produces a suitable replacement for the following argument:

(1a) Tom is thinking.

(2a) If Tom is thinking, then Tom has a mental property.

(3a) Any concrete thing that has a mental property partially psychologically resembles every other concrete thing that has a mental property.

(4a) Sarah has a mental property.

(5a) Tom and Sarah are concrete things.

(Ca) Tom partially psychologically resembles Sarah.

I have yet to find a case where the strategy fails to produce suitable paraphrases of simple sentences about intrinsic properties of concrete objects and that only contain "internal" expressions, or expressions that apply to some things in virtue of how they are intrinsically or how they are related to each other. ${ }^{27}$ The paraphrase strategy, however, does require some extra care when applied to simple sentences about extrinsic properties of concrete objects or that contain "external" (i.e. non-internal) expressions. I will now explain why, and how the paraphrase strategy can be extended to such sentences.

\section{The Paraphrase Strategy, Extrinsic Properties, and External Expressions}

Consider the following sentence: 'the Burj Khalifa is tall and thus has the property of being tall'. Since the Burj Khalifa is the tallest building in the world, that sentence is appropriate for acceptance in ordinary contexts. Under the paraphrase strategy, its paraphrase is 'there is a

\footnotetext{
${ }^{27}$ This is not surprising, for as mentioned in $\mathrm{fn} .24$, it is likely that a general proof of inferential safety can be provided for the paraphrase strategy that resembles the one Skiba provides for fictionalists.
} 
pluriverse $\square \rightarrow$ the Burj Khalifa is tall and thus satisfies every and some tallness indicator' (where a tallness indicator is an open sentence-token like $* x$ is tall*). The paraphrase seems false for two reasons. First, the Burj Khalifa would not be tall were there a pluriverse. It seems plausible that for every positive real number $>100$, it is possible for there to be a building that many feet tall. If so, then the pluriverse would contain uncountably many buildings that dwarf the Burj Khalifa in height. All things considered, it would be rather small. Second, since the Burj Khalifa would not be tall were there a pluriverse, it would not satisfy every and some tallness indicator. A thing satisfies a tallness indicator only if it is tall. So while 'the Burj Khalifa is tall and thus has the property of being tall' is appropriate for acceptance in ordinary contexts, its paraphrase looks to be false and thus not to be a good paraphrase.

Fortunately, there is a reading on which the paraphrase is true. The difficulty is that 'tall' is an external expression, and 'the property of being tall' is an extrinsic property of concrete objects. Whether an external expression applies to some things does not depend solely on how they are and how they are related to each other: it also depends on how those things are related to everything else. Likewise, whether something instantiates an extrinsic property does not depend solely on how it is, but also on how it relates to everything else. The addition of a pluriverse massively expands reality, so it is no surprise that the paraphrase gets matters wrong when it is interpreted without any restrictions. But an unrestricted reading is not the only reading available: instead, "tall" can be interpreted so that the scope of comparison is limited to the actual things. On this restricted interpretation, the paraphrase is true. Even if there were a pluriverse, the Burj Khalifa would be tall in comparison to the actual things, and thus in relation to them would satisfy every and some tallness indicator. The difficulty is resolved.

More generally, when paraphrasing simple sentences about extrinsic properties of concrete objects or that contain external expressions, to apply the paraphrase strategy successfully one must do as follows: except for inscriptions and pluriverses, restrict the domain 
of discourse to the actual things, and correspondingly alter the interpretation of all the external expressions so that their scope of comparison is limited to the actual things. We are already familiar with domain restriction, as when we recognize sentences like 'there are exactly three bottles of milk' as being true when one is speaking of the bottles of milk in one's fridge, or 'this building is tall and thus has the property of being tall' as being true when one is speaking of a small-town building that is tall in relation to its neighbors yet is short in comparison to the skyscrapers of New York City (or at least, recognize it as being true if properties exist: all would agree it is at least appropriate for acceptance in ordinary contexts). If we can recognize sentences like this as being true, we can also recognize sentences like 'there is a pluriverse $\square \rightarrow$ the Burj Khalifa is tall and thus satisfies every and some tallness-indicator' as being true when the comparison class is the actual things. The counterfactual addition of the pluriverse just forces us to introduce a new domain restriction. With this restriction in place, the paraphrase strategy applies to these sentences perfectly well. The only change is that condition (d) is dropped from PP, which excluded sentences containing external expressions from PP's scope. $^{28}$

Of course, strictly speaking, nominalists do not believe that there are such things as domains of discourse - for nominalists, "domain restriction" is merely a facon de parler for the phenomenon that has been described above. A good question is how the nominalist is to make sense of that phenomenon. But however the nominalist is to make sense of it, it is not a problem that is specially posed by the paraphrase strategy: as already noted, it occurs all the

\footnotetext{
${ }^{28}$ Note that while I do not endorse any particular conception of the intrinsic/extrinsic distinction, I take it as a constraint on any plausible construal of that distinction that if something has or lacks an intrinsic property when the domain of discourse is taken to be unrestricted, it must have or lack that property on every restricted domain as well. Thus, however the intrinsic/extrinsic distinction is understood, a sentence about properties - intrinsic or extrinsic - is appropriate for acceptance in ordinary life iff its paraphrase is true under the restricted interpretation described above. This point holds even if there is disagreement about which properties are intrinsic and which properties are extrinsic. Much the same can be said about the internal/external distinction, sentences about properties containing internal/external expressions, and their paraphrases.
} 
time in ordinary discourse. Therefore, that extending the paraphrase strategy makes use of the phenomenon does not incur any additional costs.

\section{Conclusion}

I have argued that nominalists can replace simple sentences about properties of concrete objects with counterfactuals concerning how various open sentence-tokens would be were there a pluriverse. While I have only addressed these simple sentences, counterfactuals about pluriverses have the potential to serve as the basis of broader paraphrase strategies. For example, relations may be eliminable by appealing to two-and-greater-place open sentencetokens (e.g. *x is taller than $y^{*}, * x$ gave $y$ to $z^{*}$ ). Properties of properties may be eliminable by appealing to open sentence-tokens satisfiable by open sentence-tokens (e.g. *x is an anatomical indicator*). Mathematical objects may be eliminable by counterfactualizing "megethology", a nominalization of classes in the context of Lewis' plurality of worlds. ${ }^{29}$ Counterfactuals about pluriverses should be pressed into the service of nominalism as far as possible, and we are far from reaching their limit.

\section{Acknowledgements}

I am extremely grateful to Peter van Inwagen, Cian Dorr, Daniel Nolan, Jeff Speaks, Sara Bernstein, Geoffrey Hall, Benjamin Middleton, and my anonymous reviewers for very useful comments on this manuscript and related discussion.

\footnotetext{
${ }^{29}$ See the Appendix of Lewis' Parts of Classes (1990), co-written with John P. Burgess and A.P. Hazen; Lewis' (1993) "Mathematics is Megethology"; and Chapter 7 of Nolan's Topics in the Philosophy of Possible Worlds (2002). Nolan's variation would be particularly helpful. More speculatively, Chihara's (1990) system is perhaps modifiable to fit within a pluriverse-counterfactual framework (see fn. 15).
} 


\section{References}

Benacerraf, P. (1973). Mathematical Truth. Journal of Philosophy, 70(19): 661-679.

Benardete, J.A. (1964). Infinity: An Essay in Metaphysics. London, UK: Oxford University Press.

Brogaard, B. (2004). Species as Individuals. Biology and Philosophy, 19(2): 223-242.

Chihara, C. (1990). Constructibility and Mathematical Existence. New York, NY: Oxford University Press.

Dorr, C. (2008). There Are No Abstract Objects. In J. Hawthorne, T. Sider, and D.W. Zimmerman, Contemporary Debates in Metaphysics (pp. 32-63). Malden, MA: Blackwell Publishing.

Field, H. (1989). Realism, Mathematics, and Modality. New York, NY: Basil Blackwell Inc.

Geach, P. (1980). Reference and Generality (3rd ed.). London, UK: Cornell University Press. (1962).

Ghiselin, M.T. (1974). A Radical Solution to the Species Problem. Systematic Zoology, 23(4): 536-544.

Hull, D.L. (1978). A Matter of Individuality. Philosophy of Science, 45(3): 335-360.

Lewis, D. (1986). On the Plurality of Worlds. Malden, MA: Blackwell Publishing Ltd.

Lewis, D. (1991). Parts of Classes. Cambridge, MA: Basil Blackwell Ltd.

Lewis, D. (1993). Mathematics is Megethology. Philosophia Mathematica, 1(1): 3-23.

Lewis, D. (2001). Counterfactuals. Maiden, MA: Blackwell Publishers Ltd. (Original work published 1973)

Loux, M.J. (1998). Metaphysics: A Contemporary Introduction. New York, NY: Routledge.

Nolan, D. (2002). Topics in the Philosophy of Possible Worlds. New York, NY: Routledge.

Nolan, D. (Forthcoming). It's a Kind of Magic: Lewis, Magic and Properties. Synthese, Online Access October 13, 2015.

Paul, L.A. (2017). A One-Category Ontology. In J.A. Keller, Being, Freedom, and Method: Themes from the Philosophy of Peter van Inwagen (pp. 32-61). New York, NY: Oxford University Press.

Sellars, W. (1963). Abstract Entities. The Review of Metaphysics, 16(4): 627-671.

Sider, T. (2002). The Ersatz Pluriverse. Journal of Philosophy, 99: 279-315. 
Sinhababu, N. (2008). Possible Girls. Pacific Philosophical Quarterly, 89: 254-260.

Skiba, L. (Forthcoming). Fictionalism, the Safety Result and Counterpossibles. Analysis, Online Access June 25, 2019.

Williamson, T. (2017). Counterpossibles in Semantics and Metaphysics. Argumenta, 2(2): 195226.

Woodward, R. (2010). Fictionalism and Inferential Safety. Analysis, 70(3): 409-417.

Van Inwagen, P. (2004). A Theory of Properties. In D. Zimmerman (Ed.), Oxford Studies in Metaphysics, Volume I (pp. 107-138). New York, USA: Oxford University Press.

Van Inwagen, P. (2011). Relational vs. Constituent Ontologies. Philosophical Perspectives, 25: 389-405.

Van Inwagen, P. (2014). Inside and Outside the Ontology Room. In P. van Inwagen, Existence: Essays in Ontology (pp. 1-14). New York, USA: Cambridge University Press. 